\title{
Gravitation as a thermodiffusion in the physical vacuum
}

\author{
Zahid Zakir ${ }^{1}$
}

\begin{abstract}
An influence of matter on the vacuum energy density is considered and a treatment of gravitation as inhomogeneity of quantum diffusion is developed. A treatment of quantum theory as conservative diffusion [1] is briefly presented, where quantum fluctuations of energy and momentum of a classical particle occur because of interaction with physical vacuum. The increasing of particle's mean energy at such fluctuations appears as quantum phenomena, while corresponding local decreasing of mean vacuum energy to the same value appears as gravitation. For one a particle the decreasing is extremaly small and in particle physics it can be neglected. However, when large number of particles are concentrated in a small volume, consequences of the vacuum energy decreasing become appreciable and they appear as gravitation. The diffusion treatment of quantum processes thus leads to the diffusion treatment of gravitation with natural synthesis of theories of both phenomena. New properties of inhomogeneous diffusion related by local decreasing of vacuum energy, such as slowering of fluctuations of particles with delay of intensity of processes (including redshift of frequencies), drift of particles toward slower fluctuations region and their diffusive acceleration, which is independent on mass of particles, are studied. Observable effects following from the new treatment are discussed.
\end{abstract}

PACS: 02.50.Ey, 03.65.Ta , 05.40.Jc,

Key words: quantum fluctuations, diffusion, gravitation, curvature, Newtonian potential

\section{Content}

1. Particle's energy in diffusion treatment of quantum mechanics

1.1. Conservative diffusion of a light particle in a heavy particle medium ..........................35

1.2. Quantum mechanics as theory of diffusion of classical particle in vacuum................38

2. Inhomogeneous conservative diffusion and analogues of gravitational effects .........40

2.1. Energy conservation in particle+vacuum system and vacuum energy decreasing ........40

2.2. Thermal diffusion and the effect of concentration in cold region .................................4 41

2.3. Cooling of regions with a large number of light particles ....................................... 43

2.4. A cluster of light particles as an attraction centre for other light particles ................. 44

2.5. Diffusion acceleration and diffusion equivalence effect ........................................... 44

2.6. Diffusive delay of rate of intensity of processes ........................................................45

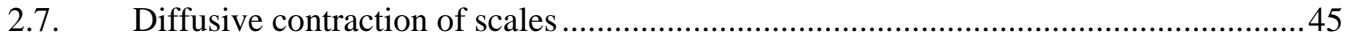

3. Gravitation as inhomogeneous conservative diffusion in vacuum ............................46

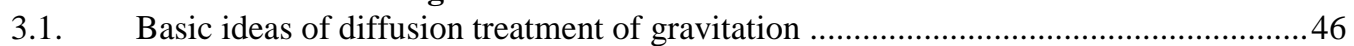

3.2. Diffusive derivation of simplest gravitational potentials ...........................................46

3.3. A physical meaning and normalization of the gravitational potential ........................ 48

3.4. Effective metrics induced by inhomogeneous diffusion ......................................... 49

3.5. Diffusion induced curvature and derivation of the field equations ..............................51

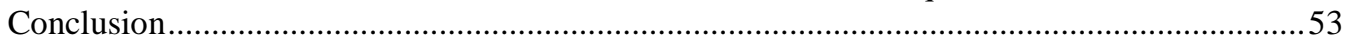

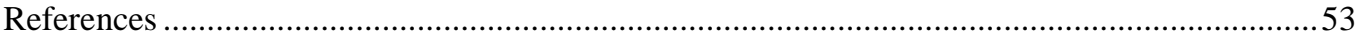

${ }^{1}$ Centre for Theoretical Physics and Astrophyics, Tashkent, Uzbekistan; zahidzakir@theor-phys.org 


\section{Introduction}

In diffusion treatment of quantum theory, described in the previous paper [1], quantum fluctuations of energy and momentum of a classical particle are considered as occurring due to an interaction with physical vacuum. At any moment of time an increasing of particle's energy then is compensated by corresponding decreasing of energy of physical vacuum and vice versa.

At any moment of time the increasing of particle's energy is compensated by decreasing of the physical vacuum energy level to the same value and vice versa. For one a particle the decreasing is extremaly small and in particle physics usually one can neglect it. However, when sufficiently large number of particles is concentrated in a small volume, or energy of fluctuations of one a particle become extremely large, consequences of the vacuum energy decreasing should be already appreciable and quite observable.

At the know high precision of quantum-mechanical calculations and measurements such local change of vacuum energy could not be remains undetected. In the present paper it will be developed a point of view that this vacuum energy decreasing really leads to a set of observable consequences which is well known and is called as gravitation. Thus, it will be shown that the diffusion treatment of quantum mechanics naturally leads to the diffusion treatment of gravitation.

For this purpose at first new properties of inhomogeneous conservative diffusion are studied in comparison with properties of usual dissipative diffusion. As a result of the local vacuum energy decreasing, in this region the intensity of fluctuations of particles decreases, which leads to delay of rate of all quantum processes, frequencies will decrease (it occurs a «redshift») and proper times will be slowed down. As a result, particles from regions with faster fluctuations will drift to this region of slower fluctuations, speed of drift will increase with each shift and there appears a diffusive acceleration which does not depend on mass of an accelerating particle. All this are characteristic properties of gravitation which in diffusion treatment is considered as manifestation of inhomogeneity of conservative diffusion in vacuum.

Up to now microscopic mechanisms of gravitation remained unknown. The Newtonian theory of gravitation was a phenomenological theory. General relativity (GR) essentially restricted a group of admissible mechanisms by exception those of them which can not enable to reduce gravitation to geometry. However, GR also does not contain guidelines about the mechanism leading to deformation of spacetime geometry in the presence of matter. Moreover, GR does not take into account the quantum phenomena which entered into physics new components - the vacuum and energy of its fluctuations.

The new diffusion treatment of gravitation initially is based on quantum conceptions and thus it realizes a surprisingly close synthesis of gravitation and quantum theory. From two basic hypotheses of modern physics - quantum fluctuations and gravitation - the diffusion treatment leaves as a hypothesis only the first, and the second makes as its consequence, i.e. gravitation appears as a part of the quantum theory.

In Chapter 1 of the paper a short review of diffusion treatment of quantum mechanics is presented, in Chapter 2 inhomogeneous conservative diffusion and analogues of gravitational effects are studied and in Chapter 3 the diffusion treatment of gravitation is described and its observable consequences are discussed. 


\section{Particle's energy in diffusion treatment of quantum mechanics}

\subsection{Conservative diffusion of a light particle in a heavy particle medium}

In classical physics, depending on a mass ratio of diffunding particles and particles of the medium, there are two qualitatively different microscopic mechanisms of diffusion.

The first is a well known mechanism of the dissipative diffusion, based on the model of the Brownian motion of large and massive (with respect to atoms) particles in a dense medium of small and light particles (atoms) [2]. At any instant any diffunding particle collides with many light particles, thus its trajectory is completely stochastic (fractal) and friction (dissipation) plays an essential role. As the result, external forces can not accelerate the diffunding particles and instead of the Newton law $\mathbf{f}=m \mathbf{a}$ there is a relation $\mathbf{v}=b \mathbf{f}$ showing that the external force leads only to the mean drift speed $\mathbf{v}$.

The second mechanism, the conservative (non-dissipative) diffusion, is based on the model of motion of light and small particles in a dilute medium of massive particles [1] before reaching the thermodynamic equilibrium of the admixture with the media. This mechanism starts with standard assumptions of the ideal gas theory that a diffunding particle: a) has the size smaller of the free pass lengths $l_{D}$; b) between collisions moves along classical trajectories and c) at any case collides elastically only with a single particle of the medium. New properties are related with possibility to neglect by dissipation of energy (friction) in quite large number of collisions that takes place in a specific case when: $g$ ) mass $m$ of the diffunding particle is much less than a medium's particle mass $M$, i.e $m \ll M$.

At collision with a heavy particle in the centre-of-mass frame the light particle's speed changes direction only, while its magnitude and kinetic energy remain practically unchanged. The trajectory of a light particle consists of the glued together smooth pieces of free pass and an influence of external fields one can account by usual laws of the Newtonian dynamics. This is one of main differences of conservative diffusion from the usual Brownian motion where such classical pieces are absent and small pieses of trajectory should be smoothed artificially.

The standard kinetic theory of diffusion of light gas in the heavy has been constructed for the dissipative regime when components of binary gas are in thermodynamic equilibrium [2]. In this case thermal energies of atoms of both components are equal and consequently speeds of light atoms essentially exceed speeds of heavy, which then allows one to build the theory at the assumption about resting heavy atoms (the Lorentz gas). In this model, at the presence of a gradient of temperature, the light gas concentrates in the high temperature region [2].

In contrast with the Lorentz gas, the theory of conservative diffusion [1] deals with an initial transition state of binary gas when the light gas at practically zero temperature is injected into the warm heavy gas and still is far from the thermodynamic equilibrium. The mean kinetic energy of light particles can essentially differ on the thermal energy of medium's particles. In this model, as it is shown below, the temperature gradient concentrates the light particles in the low temperature region.

Thus, let a light particle moves in the dilute gas of massive particles. By averaging on ensemble of light particles at any moment of time $t$ we define mean time $\tau_{D}$ and mean speed $v_{D}=l_{D} / \tau_{D}$ of free pass which give us the mean momentum $p_{D}=m v_{D}$ and the mean kinetic energy $E_{D}=m v_{D}^{2} / 2$ of free pass. The mean energy of the light particle, containing the energy 
Zakir Z. (2014) Theoretical Physics, Astrophysics and Cosmology, 9, 1, TPAC: 4874-037

of fluctuations and the energy of drift (initial and gained in an external field between collisions), is conserved. Such process is statistically reversible and there is a time reversal symmetry.

The particle's mean energy conservation allows one to enter the abbreviated action $S$ for a finite section of trajectory as the sum of short classical pieces. Averagely it will be about $\Delta S \sim N S_{D}$, where $S_{D}=p_{D} l_{D}$ is an elementary abbreviated action for the medium, separating a region of classicality of trajectories from a region of stochasticity.

In the time interval $\Delta t$ the particle lies in an element of phase volume $\Delta p \Delta x$. In our case there is an elementary phase volume:

$$
\Gamma_{D}=p_{D} l_{D}=m l_{D}^{2} / \tau_{D},
$$

coinciding by $S_{D}$. Therefore it is natural to take as primary $\Gamma_{D}$, and to express through it other characteristics of the system. In particular, defining (formally) a diffusion coefficient as $l_{D}^{2}=2 D \tau_{D}$, from (1) we obtain:

$$
D=\Gamma_{D} / 2 m,
$$

i.e. $2 D$ in fact is $\Gamma_{D}$ for a particle of unit mass.

In the paper [1] the theory of conservative diffusion has been formulated not by analogy to the theory of Brownian motion, which here is inapplicable and leads to internal contradictions, but on the basis of the hydrodynamic analogy as natural to the conservative region of diffusion with a free pass length. Particle's coordinates $x(t)$ are fixed through quite small intervals of time $\Delta t \ll \tau_{D}$ when motion occurs (averagely) over the classical trajectories.

Particle's mean speed consists on the drift $\mathbf{v}$ and diffusion $\mathbf{u}$ speeds. Since particle's trajectory between collisions is classical, thus, as well as in the Hamiltonian dynamics, the drift component of momentum $\mathbf{p}_{\mathbf{v}}=m \mathbf{v}$, describing an initial speed and influence of external fields, can be taken as gradient of the action function $S(x, t)$ :

$$
\mathbf{p}_{\mathbf{v}}(x, t)=\nabla S(x, t) \text {. }
$$

For diffusion speed $\mathbf{u}$ (and momentum $\mathbf{p}_{\mathbf{u}}=m \mathbf{u}$ ), related by fluctuations, the mean over ensemble vanishes, while root-mean-square value is non-zero:

$$
\overline{\mathbf{u}}=\int \mathbf{u} \rho d^{3} x=0, \quad \int \mathbf{u}^{2} \rho d^{3} x \neq 0 .
$$

Here $\rho(x, t)$ is the probability density which is normalized and satisfy, due to probability conservation, to the continuity equation:

$$
\frac{\partial \rho}{\partial t}+\nabla \cdot(\mathbf{v} \rho)=0, \quad \int \rho(x, t) d^{3} x=1 .
$$

Vanishing of the volume integral (4) from the diffusion flux $\mathbf{u} \rho$ means that the last one is a gradient of some function $f(x, t)$ vanishing at spatial infinity for vanishing of the surface integral. Due to the normalization conditions, $\rho$ also vanishes at spatial infinity, and thus we can represent $f$ in the form $f=-\rho D$, where $D(x, t)$ is that part of $f$ which distinguishes it from $\rho$ and it may not disappear on the bondary of integration. The sign here is chosen such 
that $\mathbf{u} \rho$ on sense was analogue of the diffusion flux $\mathbf{i}_{n}=-\rho D \nabla n$ opposite directed to the gradient of concentration. Thus, a dependence between $\mathbf{u}$ and $\rho$ follows from the general properties of these functions and is determined up to a certain function $D(x, t)$ which will appear further as the diffusion coefficient. Thus, from:

$$
\overline{\mathbf{u}}=\int \mathbf{u} \rho d^{3} x=\int \nabla f d^{3} x=-\int \frac{\nabla(\rho D)}{\rho} \rho d^{3} x=-\int_{\Sigma} \rho D d \Sigma=0,
$$

we obtain an expression connecting $\mathbf{u}$ with $\rho$ :

$$
\mathbf{u}(x, t)=-\frac{\nabla(\rho D)}{\rho} .
$$

From this expression follows the uncertainty relation ( at $\overline{\mathbf{x}}=0$ ):

$$
\overline{\mathbf{p}_{\mathbf{u}}^{2}} \cdot \overline{\mathbf{x}^{2}} \geq m^{2} \bar{D}^{2} \text {. }
$$

The corresponding energy of fluctuations is:

$$
U_{u}=\frac{\mathbf{p}_{\mathbf{u}}^{2}}{2 m} .
$$

Thus, the kinetic energy of the particle is the sum of drift and diffusion parts and for the Hamiltonian we obtain the expression:

$$
H=\int\left(\frac{\mathbf{p}_{\mathbf{v}}^{2}}{2 m}+\frac{\mathbf{p}_{\mathbf{u}}^{2}}{2 m}+V\right) \rho d^{3} x,
$$

which at $D=$ const. and using (5) and (10) can be written in terms $S$ and $\rho$ as:

$$
H=\int\left(\frac{1}{2 m}(\nabla S)^{2}+\frac{m D^{2}}{2}\left(\frac{\nabla \rho}{\rho}\right)^{2}+V\right) \rho d^{3} x .
$$

In this "hydrodynamic" Hamiltonian $H$ two functions, $S(x, t)$ and $\rho(x, t)$, play the role of canonical variables. The Poisson brackets in terms of this "hydrodynamic" canonical pair have the form:

$$
\begin{aligned}
& \{A, B\}=\int\left(\frac{\delta A}{\delta \rho} \frac{\delta B}{\delta S}-\frac{\delta B}{\delta \rho} \frac{\delta A}{\delta S}\right) d^{3} x, \\
& \left\{\rho(x, t), S\left(x^{\prime}, t\right)\right\}=\delta\left(x-x^{\prime}\right) .
\end{aligned}
$$

Corresponding canonical equations:

$$
\frac{\partial S}{\partial t}=\{S, H\}=-\frac{\delta H}{\delta \rho}, \quad \frac{\partial \rho}{\partial t}=\{\rho, H\}=\frac{\delta H}{\delta S} .
$$

give the Schrödinger equation in the "hydrodynamic" form:

$$
\begin{gathered}
\frac{\partial S}{\partial t}+\left(\frac{(\nabla S)^{2}}{2 m}+V\right)-\frac{(2 m D)^{2}}{2 m} \frac{\Delta \sqrt{\rho}}{\sqrt{\rho}}=0, \\
\frac{\partial \rho}{\partial t}+\frac{1}{m} \nabla \cdot(\rho \nabla S)=0 .
\end{gathered}
$$

The density of probability $\rho$ enters to this system of equations non-linearly, but the equations can be linearized by canonical transformations to the new canonical pair $\psi^{*}, \psi$ : 


$$
\psi=\sqrt{\rho} \exp (i S / 2 m D), \quad \psi^{*}=\sqrt{\rho} \exp (-i S / 2 m D),
$$

which transform (14)-(15) to the usual Schrödinger equation for the "wave function" $\psi(x, t)$ :

$$
i(2 m D) \frac{\partial \psi}{\partial t}=\left(-\frac{(2 m D)^{2}}{2 m} \Delta+V\right) \psi .
$$

Here it takes place the superposition of states: $\psi=c_{1} \psi_{1}+c_{2} \psi_{2}$ and, consequently, at conservative diffusion one should add not probabilities of two alternatives, but their probability amplitudes. To the Markov condition satisfy only the transition probability amplitudes, which are complex valued and allows one to formulate the theory in terms of path integrals. The physical sense of wave behaviour consists in periodic repetition along a trajectory of sites of free pass with mean length $l_{D}$, and also available the related elementary phase volume $\Gamma_{D}$.

Thus, the conservative diffusion in classical systems, at the conditions mentioned in the beginning of the section, is described by the formalism of quantum mechanics with replacement $\hbar \rightarrow 2 m D$. In fact the situation is contrary and quantum mechanics appears as a special case of the classical conservative diffusion in the physical vacuum with $2 m D=\hbar$, and thus $D=\hbar / 2 m$. From this it follows that the known quantum effects should be inherent and for other cases of conservative diffusion at $D \neq \hbar / 2 \mathrm{~m}$. The conclusion following from this is that in classical systems, where such diffusion would be realised, there should be also analogues of quantum effects or quasi-quantum effects [1]. Particularly, in such classical systems there should appear interference effects and wave properties for probability distributions, discreteness of energy levels and angular momenta, also should be effects of quantum statistics.

\subsection{Quantum mechanics as theory of diffusion of classical particle in vacuum}

a) The nonrelativistic theory and energy of localization.

Quantum mechanics, as it is clear from (17), appears as a special case of conservative diffusion of classical particles in the physical vacuum, when the elementary phase volume is equal to the Planck constant $\Gamma_{D}=\hbar$. Then the diffusion coefficient $D=\hbar / 2 m$ appears as a constant depending on particle's mass only. This means that the influence of vacuum on classical particles of quite small mass leads to their specific diffusive behaviour with some free pass length and conserving mean energy [1].

"Quantization" then is reduced to the feature, that a classical particle, which in the nonrelativistic theory in classical (empty) space and potential $V$ of an external force would have total energy $\mathbf{p}_{\mathbf{v}}^{2} / 2 m+V$, being placed in the physical vacuum interacts with it and starts to fluctuate, thus there appear the fluctuational contributions to its momentum and energy. The energy of such fluctuations we will denote as $U$.

The state of a free particle usually is represented as a plane wave

$$
\psi=\text { const } \cdot \mathrm{e}^{i(\mathbf{p x}-E t) / 2 m D}
$$

where $S(\mathbf{x}, t)=\mathbf{p x}-E t$ and $\rho=$ const., so in this case $\mathbf{u} \sim \nabla \rho=0$ and there is no diffusion part of the nonrelativistic momentum $\mathbf{p}_{u}=0$, and thus $E_{u}=0$. Here $U=U_{0}=$ const . and the probability amplitude of the particle is "smeared" over entire space 
and interaction with vacuum is appear as a "wave" behaviour of this amplitude, when energy and an momentum of drift are expressed through the frequency and wave vector.

For a bounded state $S(t)=-E t$ and $\rho(x)$ provides particle's localisation at a vicinity of the centre of inertia with $\mathbf{u}^{2}>0$. It testifies that the speed $\mathbf{u}$, playing the role of diffusive flux, and corresponding energy $U_{u}$ are related with particle's localisation in a finite volume and that the last one is that part of $U$ which has been transferred to the particle at narrowing of its probability distribution (by means of walls of a box or a force etc.). The part of energy of fluctuations of a particle $U$, which has been generally "smeared" on entire space, at restriction of particle's free diffusion concentrates in smaller volume. Thus, as a localisation region is less, as high the localisation energy $U_{u}$, and this shows the uncertainty relation (8).

\section{b) Relativistic theory and the rest energy.}

At foundation of the relativistic theory it has been entered into physics a mysterious constant addition to energy of any finite mass particle, the rest energy $E_{0}=m c^{2}$, which then has been checked experimentally. In the quantum theory the rest energy appeared in connection with not less mysterious circumstances - when discovered the scalar equation, considered the rest energy as an artifact of fourth spatial dimension (which then has been forgotten), while when discovered the spinor equation, has obligated to enter antiparticles mainly in connection with the rest energy. In all three cases inevitability of appearance of this energy in a formalism was supplemented by lack of any physical mechanism or a source for it. Up to now in the standard paradigm a meaning of the rest energy did not become clearer and, as well as charges of fundamental fields, it is taking as the fact.

The diffusion treatment naturally leads to the rest energy and, consequently, clears up an origin of this energy. Really, at any diffusion a part of the medium's energy is paid out for fluctuations of a diffunding particle and this energy is proportional to particle's mass. Thus, at diffusion treatment of quantum theory we should take into account that for quantum fluctuations of any particle the vacuum also "spends" an energy $U \sim m$.

As it was already discussed, the total energy of fluctuations $U$ can contain the localisation energy $U_{u}$, which is present in the nonrelativistic theory too, but is absent for free particles. Therefore, if we exclude $U_{u}$ from $U$ and also turn to a rest frame of the particle for excluding the drift speed's relativistic kinematic corrections, the particle do not cease to interact with vacuum and the energy of fluctuations shold not fully disappear at such changing of the external conditions. Therefore, as well as at any diffusion process, in any case here it should remain some constant residual component of the fluctuation energy equal to

$$
U_{0}=m \varphi_{0} .
$$

Notice, that this constant energy $U_{0}$ should exist for localised and free particles, moving and resting ones. Such part of particle's energy should appeared earlier both in the theory and in experiment, and in the former treatments, not including vacuum as an active participant of processes, it should look as unexplainable and mysterious.

The unique known part of energy of particles, obeying to these requirements and having with the same properties is the rest energy $E_{0}$ of relativistic theory and, thus, we have 
Zakir Z. (2014) Theoretical Physics, Astrophysics and Cosmology, 9, 1, TPAC: 4874-037

a right to identify this part of energy with the constant part of the energy of fluctuations $U_{0}=E_{0}$. It allows one to define a constant in (19) as in relativistic kinematics: $\varphi_{0}=c^{2}$.

Thus, the energy of fluctuations of the finite mass particle, due to the influence of physical vacuum, contains a constant part equal to the rest energy:

$$
U_{0}=m \varphi_{0}=m c^{2} .
$$

Here the physical vacuum appears as a certain fluctuating field with mean potential $\varphi_{0}=c^{2}$, where the role of a "charge" plays the particle's rest mass. Continuing this analogy we can interprete $U_{0}$ as an energy level which the particle occupies in the "vacuum field" $\varphi_{0}$.

The considered treatment of the one particle diffusion seems as a simple renaming not changing observable consequences. However, further it will be shown that for systems of diffunding particles a logical development of this picture leads to non-trivial observable consequences, allowing one to understand some phenomena earlier remained unclear.

\section{Inhomogeneous conservative diffusion and analogues of gravitational effects}

\subsection{Energy conservation in particle+vacuum system and vacuum energy decreasing}

In the previous part of the paper motion of single light classical particle in the medium of heavy particles was considered. Because of statistical description it was necessary to suppose an ensemble of light particles which can be realised by many times repetition of a situation with one particle with the same initial momentum. In this case a backward action of one particle on properties of the medium was extremaly small and it was neglected. Nevertheless, the fact of existence of such backward action in a diffusion picture has significant importance.

In the former treatments of quantum mechanics, assuming smoothness of space and excluding activity of vacuum, all features of quantum behaviour of particles were attributed to particles, entering special "quantum particles", essentially distinct from the classical ones. As a result, the fact of quantum fluctuations led in them to a problem of non-conservation of energy and momentum of particles at microscopic scales and for small time intervals.

In diffusion treatment the energy conservation requirement in the system particle+vacuum, as well as at any diffusion process, is satisfied strongly. At any moment of time the increasing of particle's energy is compensated by corresponding local decreasing of the energy of physical vacuum and vice versa. The vacuum energy in some volume, equal to any value $E_{v}$ at lack of a particle, after "inserting" of the particle decreases (in entire space) on the average value of fluctuation energy of this particle $E_{v}-U$.

In this part of the paper we will consider further simultaneous presence at small volume of quite large number of light particles $N \gg 1$ when their joint influence on the medium becomes essential and we will consider consequences of such influence.

At introduction of $N$ particles the total energy of the medium in entire volume decseases at least to the value $N \cdot U$, becoming equal to $E_{v}-N \cdot U$. Therefore, if the vacuum's energy is lowered to $U_{0}=m c^{2}$ due to quantum fluctuations of each free particle, as 
it is accepted in the diffusion treatment of quantum theory, then the presence in some volume of $N$ such particles lowers the vacuum energy at least to the value $N m c^{2}$.

The balance of energies in the system "particle+medium" gives only total change of energy of the medium, but generally does not allow one to define a spatial distribution of local energy levels of the medium. For definition of such distribution some additional conditions are required. Notice, that it is necessary to distinguish an intuitively clear picture of random walk of one particle with unique, though very disordered, a trajectory, from the probabilistic description of diffusion of ensemble of such particles. The latter one is based on the "hydrodynamic" functions $S(x, t)$ and $\rho(x, t)$ which at any moment are distributed on entire space. The changing of vacuum energy levels is defined by these functions - if the energy of the ensemble is distributed with probability density $\rho(x, t)$, than the probability of vacuum energy decreasing in each point should correlate with this distribution.

At the presence of many light particles the energy density of vacuum depends on the probability density of the system of particles as a whole. Since the last one depends also on a relative location of the particles, for different configurations there will be also different distributions of the vacuum energy density. In general case this factor complicates the situation, but at the existence of location symmetries it will allow to simplify solution of the problem, which will be considered in the Part 3 of the papers.

However, already general properties of inhomogeneous conservative diffusion, which will be considered below, follow a number of in principle new consequences, such as:

a) concentration of light particles in a cold region;

b) cooling of the medium in regions with large number of light particles;

c) a concentration region of light particles acts as an attraction centre for light particles;

d) diffusive acceleration of light particles to this region;

e) independence of such acceleration of mass of a particle;

f) relative delay of all processes in the cold region.

Already these properties reveal deep relation of inhomogeneous diffusion with such fundamental phenomenon as gravitation and appear as analogues of gravitational effects.

\subsection{Thermal diffusion and the effect of concentration in cold region}

The kinetic theory of diffusion of light gas in heavy has been formulated earlier for a case when light gas already is in thermodynamic equilibrium with heavy gas. Mean thermal energies of heavy and light atoms are equal in such mix and thermal speed of light atoms is sufficienly larger than for heavy, so one can consider the latter as rested (the Lorentz gas):

$$
\frac{m}{2} \overline{\mathbf{v}^{2}} \simeq \frac{M}{2} \overline{\mathbf{V}^{2}}, \quad \overline{\mathbf{v}^{2}} \simeq \frac{M}{m} \overline{\mathbf{V}^{2}} \gg \overline{\mathbf{V}^{2}},
$$

where $\mathbf{V}, M$ - speed and mass of heavy atom.

The theory of conservative diffusion is appropriate to a short interval of time when light gas with almost zero temperature begins to diffund in warm heavy gas and still it is still far from thermal equilibrium with the latter. In this transition period an initial energy of drift of light atoms approximately conserves and here the situation is opposite to the Lorentz gas. Now light gas initially can be considered as almost rested $\mathbf{v}_{0} \ll \mathbf{V}$, and then the collisions with the heavy atoms do the thermal speed of light atoms almost the same as for the heavy:

$$
\overline{\mathbf{v}^{2}} \simeq \overline{\mathbf{V}^{2}} \text {. }
$$


Zakir Z. (2014) Theoretical Physics, Astrophysics and Cosmology, 9, 1, TPAC: 4874-037

This means that the light gas, be slightly warmed-up due to collisions, nevertheless remains sufficiently colder than the heavy: $T_{l} \approx T_{h} m / M \ll T_{h}$.

Really, in the rest frame of a heavy atom at elastic collision of a light atom with heavy the magnitude of light atom's speed practiclly does not change $|\mathbf{v}| \simeq\left|\mathbf{v}^{\prime}\right|$, and at transition to the laboratory system, where the container with gas is rested, to the light atom's speed it should be added the speed of heavy: $\mathbf{v}^{\prime}=\mathbf{v}+\mathbf{V}$. Therefore if an initial speed has been choosen as small $\left|\mathbf{v}_{0}\right| \ll|\mathbf{V}|$, further the light atoms will have the same thermal speed as heavies $\mathbf{v}^{\prime} \approx \mathbf{V}$ and due to small mass the diffunding light gas remains sufficiently colder than the heavy:

$$
\frac{m}{2} \overline{\mathbf{v}^{2}} \ll \frac{M}{2} \overline{\mathbf{V}^{2}}, \quad T_{l} \ll T_{h} .
$$

Let in the mixture of light gas in heavy there is a temperature gradient $\nabla T \neq 0$ and $T$ decreases along $x$ axis $\nabla_{x} T<0$, i.e. at the right side the heavy gas is colder, than at the left. In usual kinetics of binary gas [2] the arising thermal diffusion flux is taken in the form

$$
\mathbf{i}_{T}=-\rho D k_{T} \cdot \frac{\nabla T}{T},
$$

where $D k_{T}$ is a thermal diffusion coefficient. This flux generates a gradient of concentration of light gas $\mathbf{i}_{n}=-\rho D \cdot \nabla n_{l}$ and there appears the opposite directed diffusion flux smoothing the concentration. Thus, the total flux of the light atoms $\mathbf{i}$ contains two opposite directed contributions and the expressions for it have the form:

$$
\begin{aligned}
& \mathbf{i}=-\frac{1}{3 N} \nabla\left(\frac{n}{T}\left\langle\frac{v}{\sigma_{t}}\right\rangle\right), \\
& \mathbf{i}=\mathbf{i}_{n}+\mathbf{i}_{T}=-\rho D\left(\nabla n_{l}+k_{T} \frac{\nabla T}{T}\right),
\end{aligned}
$$

where $\sigma_{t}$ is the transport cross section of collisions and averaging is on speed $v$. At approaching of equilibrium between two fluxes $\mathbf{i}_{n}=-\mathbf{i}_{T}$, when total flux vanishes $\mathbf{i}=0$, Eq. (25) gives:

$$
n_{l}=\operatorname{const} \frac{T}{\left\langle v / \sigma_{t}\right\rangle} \sim T \text {. }
$$

This means that in such mixture the light gas concentrates in regions with high temperature. This effect is often used in practice, in particular, at separation of isotopes.

In this model of the equilibrium mixture the thermal speed of light atoms is sufficiently larger than of heavy and a small difference of thermal speeds of heavy atoms from the right and left hand sides is extremaly small in comparison with the thermal speed of the light atoms. As a result, the direction of drift of light atoms is defined mainly by a difference of densities of the medium at the left and the right hand sides, and the high speed light atoms will be simply pressed out from the cold and denser regions to the warmer and less density regions.

In our case of conservative diffusion here occurs an opposite effect which can be easily understanded qualitatively. On any surface between warmer and colder regions the mean speed of heavy atoms coming from the warm side is higher than of atoms coming from the cold side. 
The arising nonzero difference of thermal speeds of the light atoms, collided with them near the surface, make an appreciable amount of their thermal speed and leads to thermal diffusion flux of light atoms toward the cold region. Due to the low temperature of the light gas, the thermal flux becomes stronger, while the opposite directed flux, smoothing the concentrations, becomes weaker. Thus, for the light gas the thermal flux plays the dominant role and the light gas concentrates in the region with low temperature.

Let's consider a quantitative estimation of this effect. At conservative diffusion, as well as at usual Brownian motion, we deal with the description of random walk of one a particle and instead of the concentration $n(x, t)$ there figures the probability density $\rho(x, t)$ in the ensemble, and instead of the diffusion flux $\mathbf{i}_{n}$, smoothing consentrations, figures $\rho \mathbf{u}$ (see Eq. (7)) (which further we will denote as $\rho \mathbf{u}_{\rho}$ ) smoothing the probability density. But at the presence of a temperature gradient, there appears a thermal diffusion flux proportional to $-\nabla T$ and average of total speed of diffusion $\mathbf{u}$ becomes non-zero. Then, as in Eq. (25), we can write:

$$
\rho \mathbf{u}=\rho\left(\mathbf{u}_{\rho}+\mathbf{u}_{T}\right)=-\rho D\left(\frac{\nabla(\rho D)}{\rho D}+k_{T} \frac{\nabla T}{T}\right)
$$

which gives:

$$
\mathbf{u}=-D \cdot \nabla\left[\ln \left(\rho D T^{k_{T}}\right)\right] .
$$

If inhomogeneity of $\rho$ and $D$ is caused by the temperature gradient, the flux $\mathbf{u}_{\rho}$ remains weaker than the thermal flux $\mathbf{u}_{T}$, i.e. $\nabla\left(\ln T^{k_{T}}\right) \gg \nabla \ln (\rho D)$, and the total flux is reduced to the thermal diffusion flux directed toward the cold region. It is evident in simplest case when an initial state is a "flat wave" (18) and $\mathbf{u}_{\rho}=0$. In this example further the dominating thermodiffusion forms a peak in the distribution of $\rho$ near the cold wall and $\mathbf{u}_{\rho}$ only expand the thickness of this peak up to few free pass lengths which is usually very small in comparison with the size of the several orderds larger region of conservativity.

Thus, in the conservative diffusion the light gas concentrates in the region with low temperature, which represents a new effect allowing one, by creating corresponding conditions, to check the theory in experiment.

Notice, that in process of approach of thermodynamic equilibrium the light gas heats up to the temperature of heavy and at such growth of temperature the thermal diffusion is weakened, while the smoothing concentrations flux amplifies. After some time both fluxes become balanced which then leads to usual effect of the Lorentz gas.

\subsection{Cooling of regions with a large number of light particles}

In the previous section an influence to light particles the existence of a temperature gradient was considered independently on an origin of such varying of temperatures at different regions. Here we will consider a special case when the gradient of temperature is generated not by external reasons, but due to an influence to the medium of the light particles by itself.

Let in a volume with heavy gas the concentration of light atoms $n_{l}{ }^{\prime}$ near the right wall is sufficiently higher, than their concentration $n_{l}$ near the left wall. Then near the right wall at any time interval there occur sufficiently larger number of collisions of the light atoms with the heavy, than near the left wall. Therefore, that part of thermal energy of the medium which is 
Zakir Z. (2014) Theoretical Physics, Astrophysics and Cosmology, 9, 1, TPAC: 4874-037

paid out for heating the light gas up to temperature $T_{l}$ from (23) on the right side is more than at the left side and this leads to lower medium's local temperature on the right side $T_{h}>T_{h}{ }^{\prime}$.

Thus, in region with high concentration of light gas temperature of the medium becomes lower, than in regions with smaller concentration of light atoms and an appeared temperature gradient is proportional to initial gradient of concentration, but opposite directed:

$$
\nabla T \sim-\nabla n_{l} \text {. }
$$

As a result, in the region with high concentration of light atoms the thermal speed of heavy atoms considerably decreases, and then the thermal speed of light atoms decreases too:

$$
\overline{\mathbf{V}^{2}}>\overline{\mathbf{V}^{\prime 2}}, \quad \overline{\mathbf{v}^{2}}>\overline{\mathbf{v}^{\prime 2}}, \quad n_{l}<n_{l}{ }^{\prime} .
$$

\subsection{A cluster of light particles as an attraction centre for other light particles}

Let an initial temperature if the heavy gas is the same everyvere, but an initial concentration of light particles $n_{l}\left(x, t_{0}\right)$ near the right wall is sufficiently higher than the concentration near the left wall and $\nabla n_{l}>0$. As it was discussed in the previous Section, this inhomogeneity generates immediately an opposite directed gradient of temperature of the heavy gas $\nabla T<0$ and at the right the temperature becomes lower, than at the left $T_{h}>T_{h}{ }^{\prime}$.

At the same time, as it was discussed in the section 2.2, at the presence of the temperature gradient there appears the thermal diffusion flux $\mathbf{u}_{T}$ of the light particles Eq. (24) , proportional $\nabla T$ and directed to the region with lower temperature.

As the result, from the one side, high concentration of the light particles leads to cooling of its concentration region and, from the other side, this region, due to its lower temperature, effectively attracts the light particles from lower concentration and higher temperature regions.

In fact, if one will abstract from the medium and will observe only by the light particles, then the process appear as if the cluster of light particles effectively plays the role of an attraction centre for other light particles and the speed of "falling" of latters to this cluster is increases by increasing the mass of this cluster.

\subsection{Diffusion acceleration and diffusion equivalence effect}

The presence of the temperature gradient, i.e. the thermal speed of heavy atoms, leads to the same gradient of the thermal speed of light atoms:

$$
\begin{aligned}
& \overline{\mathbf{V}^{2}(x-\Delta x)}>\overline{\mathbf{V}^{2}(x)}>\overline{\mathbf{V}^{2}(x+\Delta x)}, \\
& \overline{\mathbf{v}^{2}(x-\Delta x)}>\overline{\mathbf{v}^{2}(x)}>\overline{\mathbf{v}^{2}(x+\Delta x)} .
\end{aligned}
$$

In a volume of thickness $\Delta x$ at the left hand side of some surface the thermal speed of the light atoms changes at collision with heavy atoms more than in some volume at the right hand side and there is uncompensated difference.

The increments of drift speed of light particles, gain due to such difference, conserves throughout the conservative diffusion region. Therefore, the additional increments at further shifts accumulate and there appears the diffusion acceleration $a_{D}(x)$ directed from left to right, i.e. from warm to colder region. This acceleration is related by gradient of square-root speed: 


$$
a_{D}(x)=\frac{1}{\Delta x}\left(\overline{\mathbf{v}^{2}(x-\Delta x)}-\overline{\mathbf{v}^{2}(x)}\right)>0 .
$$

This acceleration, thus, is proportion to the temperature gradient:

$$
a_{D}(x) \sim-\nabla T \text {. }
$$

As the result, at inhomogeneous conservative diffusion the light particles not only move by a drift speed, as at ordinary diffusion, but also are accelerated toward the colder region.

Let's consider one more new and very important property of inhomogeneous conservative diffusion - independence of acceleration on mass of an accelerated light particle.

At inhomogeneous diffusion in the Lorentz gas, due to thermal equilibrium with medium, both thermal speeds and diffusion accelerations of light atoms depend on their masses:

$$
\overline{\overline{\mathbf{v}_{1}^{2}(x)}} \sim \frac{m_{2}}{m_{1}^{2}(x)}, \quad \frac{a_{1}(x)}{a_{2}(x)} \sim \frac{m_{2}}{m_{1}} .
$$

At conservative diffusion, as it follows from Eqs. (22) and (33), the thermal speeds of light particles are the same as for heavy particles of the medium and for this reason their accelerations do not depend on their masses:

$$
\frac{\overline{\mathbf{v}_{1}^{2}(x)}}{\overline{\mathbf{v}_{2}^{2}(x)}} \simeq \frac{\overline{\mathbf{V}^{2}(x)}}{\overline{\mathbf{V}^{2}(x)}}=1, \quad \frac{a_{1}(x)}{a_{2}(x)} \simeq 1 .
$$

Especially due to the elastic collisions and equity of thermal speeds of diffunding particles and medium's particles, the diffusion accelerations of the diffunding particles of different mass are equal and further this property we will call as the diffusion equivalence effect.

Thus, independence on mass of diffusion acceleration of light particles is specific property of conservative diffusion and this new effect also can be checked in experiment.

\subsection{Diffusive delay of rate of intensity of processes}

Decreasing of thermal speed in colder region leads to decreasing of diffusion coefficient in this region and to decrease intensity of all phenomena related with fluctuation of speed of a light particle. In particular, in the "diffusion wave function" of an ensemble of light particles, as in Eq. (18), frequencies decrease and wave lengths increase.

Such diffusion delay of rate of all quasi-quantum processes in a mixture of gases with "redshift" of the frequencies, compared to their energy levels, should take place in the diffusion treatment of quantum mechanics too. In this case a large concentration of particles in a small region of space leads to appreciable decreasing of the local vacuum energy level and to delay of intensity of quantum fluctuations with decreasing of all frequencies.

This diffusive delay of rate of fluctuations then is interpreted by a distant observer as delay of proper times of light particles in their high concentration region. This effect of diffusive delay of fluctuations in application to quantum processes is completely similar to the gravitational delay of proper times.

\subsection{Diffusive contraction of scales}

Decreasing of thermal speed in colder region leads also to reduction of the free pass length and increasing of density of the medium and clusters of light particles. From outside all these look as contaction of scales.

Classical example of changing of scales in condensed media is the change of volumes with temperature. At conservative diffusion the properties of the medium remain the same so these examples belong to our case also. 
Zakir Z. (2014) Theoretical Physics, Astrophysics and Cosmology, 9, 1, TPAC: 4874-037

The extrapolation of these effects on the physical vacuum at the diffusion treatment of quantum phenomena looks as slightly unusual. However the history of quantum theory, almost always created problems with evident representation of described effects, in this case most likely gives an opposite example. Now it looks unusual the existence of so simple physical explanation of such puzzle phenomenon, as contraction of lengths in gravitational field.

\section{Gravitation as inhomogeneous conservative diffusion in vacuum}

\subsection{Basic ideas of diffusion treatment of gravitation}

At conservative diffusion a large concentration of diffunding particles decreases energy of the medium in this region which then reduces intensity of fluctuations. As a result, particles from other regions with faster fluctuations will drift toward the region of slower fluctuations with growing speed of drift and this acceleration is independent on mass.

All these are characteristic properties of gravitation which allow one, starting from the diffusive nature of quantum phenomena [1], to develop the diffusion treatment of gravitation, considering gravitation as manifestation of inhomogeneity of this quantum diffusion, i.e. as inhomogeneous of conservative diffusion of particles in physical vacuum. Earlier attempt to formulate such treatment see in the papers [3].

Such model of diffusive gravitation will represent in fact the accounting of consequences of energy conservation in the "particle+vacuum" system, that during quantum fluctuations at each random increasing of particle's energy a local vacuum energy decreases to the same value and vice versa. Here the rest energy of a finite mass particle appears as a part of an energy of its quantum fluctuations and also should be taken into account in the balance of energies. Then a magnitude of decreasing of the «energy level» of vacuum in each point, after averaging in time, is treated as the gravitational potential created by this particle in this point.

The gravitational energy then is appear as a local deficit of the vacuum energy which has arisen because the part of vacuum energy has passed to a particle as mean energy of its quantum fluctuations, including ordinary fluctuations and the rest energy. This fact will be clearly evident at a diffusive derivation of simplest gravitational potentials in the next Section.

The described diffusive treatment naturally explains also that fact that gravitation acts as a change of spacetime geometry. In GR this fact has been postulated, whereas in the diffusive approach the effective metrics and effective curvature of spacetime follow from the nature of behaviour of particles and their world lines moving in inhomogeneously fluctuating vacuum. These questions will be considered in Sections 3.3 and 3.4.

\subsection{Diffusive derivation of simplest gravitational potentials}

If gravitation is diffusion, by consideration of simplest symmetric configurations of light particles one should come to the same experimentally found gravitational potentials which then have been derived in GR geometrically. Let us consider this problem below.

\section{a. Homogeneous potential between two plates}

Let two thin and flat dust plates of area $S$ are rested at large distance and the rest energy of $N$ particles of each of plates is equal to $E_{0}=\bar{M} c^{2}$, where $\bar{M}=N m$. Between the plates the vacuum energy decreasing due to fluctuations of the particles is deeper than outside of this region. Thus, with respect to the external regions the internal region appears as a potential well. 
For this reason the fluctuations of particles of each plate become asymmetric - in the exterior they are more intensive than in the interior. This leads to slow drift and acceleration of each of particles of both plates toward to other plate.

At drift on $\Delta x$ a part of vacuum energy fluctuations in internal region it is spent for giving of speed of drift to particles and passes to the kinetic energy of plates related with speed of drift $v$. Respectively, vacuum energy decreases by value of this kinetic energy so the potential hole between plates goes deep.

At two shifts to the same value $\Delta x$ the decreasing of volumes of internal region $\Delta V=S \cdot \Delta x$ are equal too. Therefore, the vacuum energy decreasing is the same too and at any $x$ is proportional to the mass of a particle and shift $\Delta x$. As a result, at shifting to the same $\Delta x$ at two values of $x$ the relation of the kinetic energy changings is constant:

$$
\frac{v^{2}\left(x_{1}+\Delta x\right)-v^{2}\left(x_{1}\right)}{v^{2}\left(x_{2}+\Delta x\right)-v^{2}\left(x_{2}\right)}=\text { const }
$$

By introducing the acceleration as

$$
a_{0}=\frac{d v}{d t}=\frac{d x}{d t} \frac{d v}{d x}=v \frac{d v}{d x}=\frac{1}{2} \frac{d\left(v^{2}\right)}{d x}=\text { const., }
$$

we see that the proportionality constant in Eq. (37) is equal to $2 a_{0}$ and it appears as the acceleration in the positive direction from the rest at $x_{0}$ :

$$
\frac{1}{2} v^{2}(x)=a_{0}\left(x-x_{0}\right) \text {. }
$$

A potential $\varphi$ for such effective homogeneous field is:

$$
a_{0} \equiv-\frac{d \varphi}{d x}, \quad \varphi_{h}(x)=-a_{0} x .
$$

The total energy of the particle of mass $m$ on the plate, beginning to diffund from the rest $v\left(x_{0}\right)=0$, is thus equal to:

$$
E=\frac{m v^{2}(x)}{2}+m \varphi_{h}(x)=m \varphi_{h}\left(x_{0}\right) .
$$

This is nothing but as the energy of a particle in a homogeneous and constant gravitational field.

\section{$b$. The Newtonian potential of a thin spherical shell}

Let the thin spherical dust shell has rested at "infinity" and the sum of rest energies of its particles has equal to $E_{0}=\bar{M} c^{2}$. At finite values of radius at interior of the shell the decreased vacuum energy level is lower than at exterior of the shell and the interior of the shell appears as a potential well. For this reason the fluctuation of particles of the shell occer asymmetrically - at external region the fluctuations are more intensive than at interior.

This leads to radial drift of each of particles of the thin shell toward the centre. At drift to $\Delta r$ a part of vacuum energy in the internal region transforms to the kinetic energy of particles of the shell, related with the drift speed $v$. Respectively the vacuum energy level at internal region decreases to the value of this kinetic energy and the potential well becomes deeper.

The vacuum energy lowering, as in the case of plates, is proportion to mass of the shell and the shift $\Delta r$. However, now the shell's area $S(r)=4 \pi r^{2}$ decreases with decreasing the radius and thus at shrinking of the shell to $\Delta r$ a volume element which it swept is equal to: 
Zakir Z. (2014) Theoretical Physics, Astrophysics and Cosmology, 9, 1, TPAC: 4874-037

$$
\Delta V=4 \pi r^{2} \Delta r .
$$

If now to compare the growth of the shell's kinetic energy at shrinking to $\Delta r$ and following vacuum energy decreasing in the shell to the same value, one can conclude that:

a) for acceleration of the same number of particles the vacuum energy is paid out from more and more decreasing volume of internal region;

$b)$ as the result, the same value of energy, which was transformed to the kinetic energy of particles, at each step leads to larger deepening of the vacuum energy level;

c) the increasing difference of the vacuum energy levels at the same shift $\Delta r$ leads to additional strengthening of the drift speed, thus lowers the vacuum energy level even more.

As a result, the vacuum energy level decreasing becomes inverse proportional to the area of the shell at given radius with corresponding growth of slope (gradient) of the potential curve. Then the relation of changes of kinetic energy of a diffunding particle for two values of radius at drift on the same $\Delta r$ is not constant, as in the case of a homogeneous field, but is inverse proportional to the relation of swepted volumes, i.e to the areas of shells at these radii:

$$
\frac{v^{2}\left(x_{1}+\Delta x\right)-v^{2}\left(x_{1}\right)}{v^{2}\left(x_{2}+\Delta x\right)-v^{2}\left(x_{2}\right)}=\frac{r_{2}^{2}}{r_{1}^{2}},
$$

As in the case of the homogeneous field, it is also the relation of accelerations, which means that now the accelerations are inversely proportional to square of the radius. Now the diffusive acceleration is directed toward the centre and consequently will have a negative sign, and also is proportional to mass of the shell $\bar{M}$, since the decreasing of of the vacuum energy level is proportional to the number of particles and mass of each of them. Thus, taking into account (38) and (43), we obtain:

$$
a(r)=-\frac{G \bar{M}}{r^{2}} \equiv-\frac{d \varphi_{g}}{d r},
$$

where the gravitational constant $G$ in principle can be calculated and expressed through the parametres of diffusion process. Then the potential of the diffusive gravitational field arising around the shell looks like:

$$
\varphi_{g}(r)=-\frac{G \bar{M}}{r} .
$$

The total energy of a particle of mass $m$ on the shell falling from the rest at $x_{0}$ is:

$$
E=\frac{m v^{2}(r)}{2}+m \varphi_{g}(r)=m \varphi_{g}\left(r_{0}\right)
$$

Thus, the diffusion mechanism for gravitation in the nonrelativistic approach reproduces the Newtonian potential $\varphi_{g}(r)$.

\subsection{A physical meaning and normalization of the gravitational potential}

Transition to relativistic theory of diffusion gravitation assumes a certain combination of quantum, gravitational and relativistic methods. Due to obvious difficulty of it, at first it is useful to consider simple physical facts allowing one to reveal a character of required theory.

The Newton potential $\varphi_{g}(r)$ in Eq. (45) is defined up to a constant $\varphi_{g}(\infty)$ which in the nonrelativistic theory, for convenience, has been chosen as equal to zero for disappearing 
of the potential at infinity. As the result, the energy of a gravitational field, as well as the total energy of a particle rested in this field at $r_{0}$, were negative defined.

The diffusion treatment gets clarity in this question that the constant $\varphi_{g}(\infty)$ is nothing but as a value of unit mass test particle's energy level in the vacuum at spatial infinity, identified by its rest energy, i.e. $\varphi_{g}(\infty)=\varphi_{\text {vac }}(\infty)=c^{2}$. Then the gravitational potential of dust shell's static field appears as a difference between the local vacuum energy level of the unit mass test particle $\varphi_{v a c}(r)$ and the level at spatial infinity $\varphi_{v a c}(\infty)=c^{2}$ :

$$
\varphi_{\text {vac }}(r)-\varphi_{\text {vac }}(\infty)=\varphi_{\text {vac }}(r)-c^{2}=\varphi_{g}(r)
$$

Then the total energy of a distant test particle of mass $m$, as in the nonrelativistic limit of the relativistic theory, includes the rest energy and takes a form:

$$
\begin{gathered}
E=m \varphi_{v a c}(r)+\frac{1}{2} m v^{2}, \\
\varphi_{v a c}(r)=c^{2}+\varphi_{g}(r)=c^{2}-\frac{G M}{r} .
\end{gathered}
$$

As a result, now not only particle's energy, but also the vacuum potential $\varphi_{\text {vac }}(r)$ are positive everywhere outside the source.

Thus, the redefined potential $\varphi_{v a c}(r)$ on the one hand is maximal on infinity, where tends to a universal constant, and on the other hand tends to zero near the gravitational radius of any source $r \rightarrow r_{g}=2 G M / c^{2}$.

At the same time, in tho diffusion picture the intensity of fluctuations at any point expresses a local temporal rate of processes with pest particles. Therefore, the relation $\varphi_{v a c}(r)$ to $\varphi_{\text {vac }}(\infty)$, showing how the local vacuum energy level lower a normal one at infinity, is also shows delay of proper times at this point.

In geometrical treatment GR this measure it is given a temporary komponenty metrics $g_{00}(r)$. In this regard we can enter as well an effective diffusive metrics, having defined it as the relation of level of vacuum energy in this place to energy level on infinity:

$$
g_{00}(r)=\frac{2 \varphi_{v a c}(r)}{2 \varphi_{v a c}(\infty)}=\frac{2 \varphi_{v a c}(r)}{c^{2}}=1+\frac{2 \varphi_{g}(r)}{c^{2}} .
$$

More detail discussion of the induced by diffusion geometrical structures, including a metrics, will be presented in the following sections.

\subsection{Effective metrics induced by inhomogeneous diffusion}

In GR the properties of gravitational field are postulated and then possibility of their representation as geometric properties of spacetime is shown. After discussing that in the diffusion treatment the properties of the field follow from quantum mechanics, now we turn to represent them in a geometrical form.

The basis for this, as well as in GR, is the independence of diffusion acceleration from mass of a test particle, but from a rank of a postulated principle now it is reduced to the level of an effect. This effect leads to the similar acceleration of particles and their macroscopical collections, including and bases of local frames of reference. But the same acceleration of 
Zakir Z. (2014) Theoretical Physics, Astrophysics and Cosmology, 9, 1, TPAC: 4874-037

objects and local systems of reference effectively is indistingushable from the existence of a non-trivial metrics and curvature of spacetime. This means that non-uniform diffusion in physical vacuum induces an effective non-trivial metrics $g_{\mu \nu}(x, t)$ and curvature.

In the considered nonrelativistic limit of relativistic kinematics in a gravitational field, already a diffusion origin, are applicable the same standard methods of transition to the geometrical picture which were used earlier at formulation of GR (see [ ]).

The local equivalence of the phenomena in a gravitational field to the transition to an accelerated frame of reference allows to use relativistic kinematics for definition of that part of the metrics which is caused the field. In particular, since the diffusion acceleration leads to the delay of proper times, then the gravitational field should lead also to the same delay.

For a test particle moving in a static gravitational field, the Lagrangian and the action function, according to (48), look like:

$$
\begin{gathered}
L=-m \varphi_{v a c}+\frac{1}{2} m v^{2}, \\
S=-m c \int d s=\int L d t=-m c \int\left(\frac{\varphi_{v a c}}{c}-\frac{v^{2}}{2 c}\right) d t .
\end{gathered}
$$

For the line element from this follows (up to accuracy $1 / c^{2}$ ):

$$
\begin{gathered}
d s^{2}=g_{00} c^{2} d t^{2}-d \mathbf{r}^{2}=\left(c+\frac{\varphi_{g}}{c}-\frac{v^{2}}{2 c}\right)^{2} d t^{2} \simeq\left(1+\frac{2 \varphi_{g}}{c^{2}}\right) c^{2} d t^{2}-d \mathbf{r}^{2}, \\
g_{00}(r) \simeq 1+\frac{2 \varphi_{g}}{c^{2}} .
\end{gathered}
$$

For transition to exact expressions we may use the energy conservation conditions in the static field. For the total energy of particles of the freely falling dust sphere, which was rested at infinity, this condition gives:

$$
\begin{gathered}
E=m c^{2} g_{00}(r) \frac{d x^{0}}{d s}=m c \frac{\sqrt{g_{00}(r)}}{\sqrt{1-v^{2}(r) / c^{2}}}=m c^{2}, \\
g_{00}(r)=1-\frac{f(r)}{c^{2}} .
\end{gathered}
$$

Here $f(r)$ is a certain function which is equal exactly to $v^{2}(r)$ from (55), and in the limit of a weak field (54) is equal to $2 \varphi_{g}(r)+O\left(1 / c^{2}\right)$. In this approximation, taking into account a velocity dependence of the test particle's local energy, we can provide an exact accounting at least of the relativistic kinematics, which gives:

$$
E=\frac{m c^{2}}{\sqrt{1-v^{2} / c^{2}}}+\frac{m c^{2}}{\sqrt{1-v^{2} / c^{2}}} \cdot \frac{\varphi_{g}}{c^{2}}=\frac{m c^{2}\left(1+\varphi_{g} / c^{2}\right)}{\sqrt{1-v^{2} / c^{2}}} .
$$

Since existing experiments have reached accuracy only of order $\varphi_{g} / c^{2}$, more exact estimations have while only methodical interest. Nevertheless they are important for solution of fundamental problems and effects in strong fields, such as collapse and gravitational waves. 
For this reason let us clarify a relation of local frames with global coordinate systems and then we will attempt to define the radial component of the metrics too. All frames of reference we will suppose as instantly-rested under the inertial system chosen earlier and another case we will note additionally.

In an inertial frame of reference in flat spacetime the standard clocks, measuring intervals $d t$, are going synchronously everywhere, the standard scales, placed along coordinate lines, everywhere are identical and the curvature tensor is equal to zero. Locally intervals of curvilinear coordinates $d x^{\mu}$ are expressed through intervals $d x^{a}$ of a standard clock and a local Cartesian triad as

$$
d x^{\mu}=e_{a}^{\mu} d x^{a}, \quad \eta^{a b} e_{a}^{\mu} e_{b}^{v}=g^{\mu v},
$$

where $a, b=0, \ldots, 3, \mu, v=0, \ldots, 3$ and $\eta^{a b}=\operatorname{diag}(1,-1,-1,-1)$. It is natural to choose the time coordinate such that its intervals are given by the intervals of standard clock's time $d x^{0}=d t$. The spatial coordinate system simplies the description if it coincides with symmetries of the describing system. But in all practically interesting cases it is possible to choose them such that at least one of coordinates, for example $x^{1}$, can be directly expressed through the standard scales. Then the interval in the inertial system has the form:

$$
d s^{2}=d t^{2}-\left(d x^{1}\right)^{2}+g_{\alpha \beta} d x^{\alpha} d x^{\beta}, \quad \alpha, \beta=2,3 .
$$

At transition to an accelerated frame of reference, in which acceleration is directed along $x^{1}$, the local metrics changes. However, continuing to use the same system of coordinates as before, which was coincided by symmetries of the problem and where two coordinates $x^{0}$ also $x^{1}$ were physical ones, we express a new interval also in terms of former coordinates:

$$
d s^{2}=g_{00} d t^{2}+g_{11}\left(d x^{1}\right)^{2}+g_{\alpha \beta} d x^{\alpha} d x^{\beta} .
$$

Here now $g_{00}$ and $g_{11}$ express a real influence of acceleration on the standard scales and clocks: $\sqrt{g_{00}}$ shows a degree of the delay of proper times, and $1 / \sqrt{-g_{11}}$ shows a degree of the contraction of standard scales. Thus the standard scales placed along axes $x^{2}$ and $x^{3}$ remain the same as in the inertial frame.

Let $x^{1}=r$ and the particle radially falls in this field of acceleration from the rest under the inertial system at distant point and up to the present point gained the speed $v(r)$. Further the processes in the accelerated, but instantly rested frame we will describe from a local inertial frame instantly comoving to the particle. In the latter frame the effects of acceleration are absent, but there are kinematic delay of proper times and contraction of scales. Again continuing to use the former coordinates, we will obtain for the line element:

$$
d s^{2}=\sqrt{1-v^{2} / c^{2}} d t^{2}-\frac{d r^{2}}{\sqrt{1-v^{2} / c^{2}}}+g_{\alpha \beta} d x^{\alpha} d x^{\beta} .
$$

Thus, in the static field, at first, the energy conservation condition gives $g_{00}(r)=1-v^{2}(r) / c^{2}$ and, secondly, relativistic kinematics leads to $g_{00}(r)=-g_{11}^{-1}(r)$.

\subsection{Diffusion induced curvature and derivation of the field equations}

In GR the geometrical interpretation of gravitation is considered as a natural consequence of the equivalence principle taken as an empirical fact. For the derivation of the 
Zakir Z. (2014) Theoretical Physics, Astrophysics and Cosmology, 9, 1, TPAC: 4874-037

field equations it is required another empirical fact that the source of the field is the energymomentum of matter. However, even the energy-momentum of matter looks like an alien element against the background of geometrical structures of the theory, while the energy of the gravitational field at all does not appear in GR in an explicit form and it is entered only in particular cases and for special physical reasons.

The diffusion treatment appears as a next step in understanding of a nature of gravitation. From this treatment it follow, as they have been shown in the simple examples, both the equivalence principle and the generation of the field by the energy-momentum of the source. Therefore the diffusion picture should reproduce all formalism of GR, but any more not as a formal mathematical construction, but as one of macroscopical methods of description of gravitation without deepening into its microscopic mechanism. As a result of clearer physical picture, the problem of the field's energy disappears too.

At inhomogeneous diffusion, as a consequence of the equivalence effect, average trajectories of free particles are not geodesics in flat spacetime and contain some deviations from the geodesics. For their description one can enter curvilinear coordinates $x^{\mu}\left(x^{a}, t\right)$ and basis vectors $e_{\mu}^{a}$ along average trajectories of free particles, where $d x^{\mu}$ are connected with the local intervals of physical coordinates of the test particle at a point $M$ as in Eq. (58). Then inhomogeneous diffusion can be described as a homogeneous one in an effective Riemann manifold with a metric tensor $g_{\mu \nu}(x, t)$, where the geodesics are average trajectories of drift.

This allows one to enter a diffusion parallel transport of tensors in flat spacetime along the average trajectory of free drift:

$$
d e_{a}^{\mu}(x, t)=-\Gamma_{v \lambda}^{\mu} e_{a}^{\lambda} d x^{b}(t),
$$

where $\Gamma_{v \lambda}^{\mu}$ is an effective connection. Then corresponding to this connection effective Riemann curvature tensor $R_{\mu \nu \lambda \sigma}$ can be introduced by the standard way.

The diffusion treatment of gravitation then can be built as quantum mechanics in effective (pseudo) Riemann spacetime. Therefore, it is possible to use known methods of the description of diffusion in the curved manifolds.

Let us consider the second postulate of GR that gravitation is generated by the energymomentum of the source. In the diffusion theory the vacuum energy around a source is lowered on the fluctuation energy of its particles, including their rest energy and also the kinetic energy which these particles have got from the vacuum at formation of the source. The particle's total energy, as in fact it follows from the diffusion treatment, is that energy to which the vacuum energy has decreased. Therefore, the sum of total energy of particles and the value of the vacuum energy decreasing is equal to zero in entire space, which in terms of energy density $\varepsilon_{m}$ for rested particles gives:

$$
\int\left(\rho_{v a c}^{(0)}-\rho_{v a c}\right) d V=\int \varepsilon_{m} d V .
$$

The vacuum energy falling around the source and its smooth restoration at removal from it, appearing in the left part, can be described as in terms of diffusion and as in terms of gravitational potential. Instead of the potential it is possible to enter a spacetime curvature. For physical purposes the curvature is used in the form of Einstein's tensor $G_{\mu v}$, which in the diffusion treatment should be identified with the energy deficit of the physical vacuum: 


$$
\rho_{\text {vac }}^{(0)}-\rho_{\text {vac }}=\frac{1}{\kappa} G_{\mu}^{\mu}
$$

In general we cannot summarise the local energy densities since different parts of the source can have different speeds under the centre of symmetry and local clocks at different points are going non-synchronously. For this reason the energy balance should be written locally and in a tensor form, i.e. through the energy-momentum tensor of the source $T_{\mu \nu}$, which then leads to Einstein's equations:

$$
\frac{1}{\kappa} G_{\mu \nu}=T_{\mu \nu} .
$$

By choosing at each point a definite local basis with 4-speed $u^{\mu}$ on some hypersurface of simultaneity and by projecting both sides of the tensor equation (65) to this basis, at each point one can obtain a scalar equation. It allows one to integrate over entire space and obtain total energy the source and its gravitational field, which according to (63) should be equal to each other.

The purposes of the paper were mainly the physical aspects of the new treatment of gravitation. More consecutive from the formally mathematical point of view formulation and broader review of consequences will be considered in the subsequent publications.

\section{Conclusion}

Thus, inhomogeneous conservative diffusion has all those main properties which still were considered peculiar only to gravitation. Analogues of gravitational effects can be quite observable in those systems where it will be possible to realise conservative diffusion. It will allow not only to check the theory on experiment, but also enters a new class of observable effects into physics, research and application which opens new prospects.

The diffusion nature of gravitation, described in paper in general form, requires further investigations and more strong justifications. But the basic statements of the theory will grounded further on those physical pictures which have been described in the paper and which probably will not undergo further essential changes because of their simplicity, generality and availability to direct checking.

The fact that precisely quantum theory appears as that mechanism of gravitation which should will appear at once in physics and that further it is not necessary to invent anything new and squeeze it into so well fine-tuned physical picture of reality, is also not only unexpected and quite satisfactory fact, but also a doubtless achievement of the new treatment.

\section{References}

1. Zakir Z. (2014) Theor. Phys., Astrophys. and Cosmol. 9(1) 18, doi: 10.9751/TPAC.4874-036.

2. Landau L.D., Lifshitz E.M. (1981) Physical Kinetics. Pergamon.

3. Zakir Z. (2009) Theor. Phys., Astrophys. and Cosmol.,

4(1) 1, doi: 10.9751/TPAC.3000-012, (1998) arXiv:hep-th/9812254;

4(1) 7, doi: 10.9751/TPAC.3000-013; (1999) arXiv:hep-th/9906079. 